\title{
Paeonol Pretreatment Attenuates Anoxia-Reoxygenation Induced Injury in Cardiac Myocytes via a BRCA1 Dependent Pathway
}

\author{
Jifeng Zheng, ${ }^{a, b}$ Zhiyao Mao, ${ }^{b}$ Jianqin Zhang, ${ }^{b}$ Liqing Jiang, ${ }^{b}$ and Ningfu Wang, ${ }^{*, a}$ \\ ${ }^{a}$ Department of Cardiology, The Affiliated Hangzhou Hospital of Nanjing Medical University, Hangzhou First \\ People's Hospital; Hangzhou 310006, China: and ${ }^{b}$ Department of Cardiology, The Affiliated Second Hospital of \\ Jiaxing Medical University; Jiaxing 314000, China. \\ Received June 27, 2020; accepted September 14, 2020
}

Breast cancer type 1 sensitive protein (BRCA1) is a well-known tumor suppressor and its role in oxidative stress has been confirmed. The purpose of this study is to evaluate whether paeonol has a protective effect on myocardial hypoxia-reoxygenation (A/R) injury, and to explore H9C2 cells through a mechanismdependent pathway mediated by BRCA1. H9C2 cells were pretreated with paeonol $(10 \mu \mathrm{M})$ for $18 \mathrm{~h}$ before hypoxia was induced to establish a cell model of myocardial ischemia/reperfusion (I/R) injury. Use commercial kits to detect antioxidant indicators, including relative oxygen content (ROS) levels, total antioxidant capacity (T-AOC), superoxide dismutase (SOD), lactate dehydrogenase (LDH) activity, and creatine kinase (CK-MB) and nuclear factor-kappaB (NF- $\kappa$ B) activity. The cell viability was analyzed by 3-(4,5-dimethylthiazol-2-yl)-2,5-diphenyltetrazolium bromide (MTT) reduction method. Real-time fluorescent quantitative PCR was used to detect BRCA1 mRNA and protein levels. The expression levels of BRCA1, NLRP3 and ACS were determined by Western blotting. In addition, the release of interleukin (IL)-1 $\beta$ (IL-1 $\beta$ ), IL-6 and tumor necrosis factor- $\alpha$ (TNF- $\alpha$ ) was also evaluated by an enzyme-linked immunosorbent assay (ELISA) kit. The results showed that paeonol $(10 \mu \mathrm{M})$ can significantly improve the hypoxic $\mathrm{A} / \mathrm{R}$ damage of $\mathrm{H} 9 \mathrm{C} 2$ cells, and the BRCA1 expression of $\mathrm{H} 9 \mathrm{C} 2$ cells pretreated with paeonol was significantly increased before $A / R$ damage was induced. BRCA1 is widely known in breast and ovarian cancer. Our data proves that the down-regulation of BRCA1 participates in the decrease of cell viability and the decrease of CK-MB and LDH activities, and protects cells by inhibiting the production of ROS and the activation of Nod-like receptor protein 3 (NLRP3) inflammasomes and $\mathrm{NF}-\boldsymbol{\kappa} \mathrm{B}$. In conclusion, paeonol significantly improved the $\mathrm{A} / \mathrm{R}$ damage of $\mathrm{H} 9 \mathrm{C} 2$ cells induced by hypoxia through the BRCA1/ROS-regulated NLRP3 inflammasome/IL- $\beta$ and NF- $\kappa$ B/TNF- $\alpha /$ IL-6 pathways. It may be a potential drug against myocardial $I / R$ injury.

Key words paeonol; anoxia-reoxygenation injury; reactive oxygen species; breast cancer type 1 sensitive protein (BRCA1); Nod-like receptor protein 3 (NLRP3) inflammasome; nuclear factor-kappaB (NF- $\kappa$ B)

\section{Introduction}

Myocardial ischemic infarction (MI) is a major cause of mortality in cardiovascular-related diseases and is still a great challenge in clinical therapy. ${ }^{1,2}$ Reperfusion therapy such as percutaneous coronary intervention (PCI) has been the most effective strategy to restore blood flow after ischemia and to reestablish the function of cardiomyocytes. however, reperfusion aggravates the injury. It is a complex process related with various mechanisms including oxidative stress, calcium-overload and mitochondrial alterations. ${ }^{3-6)}$ Myocardial ischemia and reperfusion (I/R) lead to the increase of reactive oxygen species (ROS) production, which could give rise to cardiac cells membrane lipid peroxidation, inactivation of critical enzymes and interference with nucleic acid synthesis. Alteration of these molecular structure further triggers dysfunction of cardiac myocytes. ${ }^{7,8)}$

Paeonol is the phenolic compound derived from the widely used Chinese herb Paeonia suffruticosa (cortex moutan). ${ }^{9)}$ It has been found to possess a broad range of pharmacological properties such as anti-oxidation, anti-inflammation and anti-tumor effect. ${ }^{10,11)}$ Peaonol was shown to have the ability to confer neuroprotective function by promoting heme oxygenase-1 (HO-1) expression on 6-hydroxydopamine-induced oxidative damage in cortical neurons and protect against cerebrovascular damage. ${ }^{12)}$ However, the effect of paeonol on myo- cardial I/R injury has not been studied yet. Cardiomyocytes exposed to myocardial hypoxia-reoxygenation $(\mathrm{A} / \mathrm{R})$ in vitro experience a pathophysiological process similar to myocardial $\mathrm{I} / \mathrm{R}$ injury in vivo, and it is a well established model. This study was designed to evaluate whether paeonol could confer protection against $\mathrm{A} / \mathrm{R}$ injury and investigate its underlying mechanism.

Breast cancer type 1 sensitive protein (BRCA1) gene functions as a regulator of genome stability and is involved in DNA repair, transcription and oxidative stress. ${ }^{13-15)}$ Though BRCA1 is widely known as a tumor suppressor in breast cancer, recent reports indicated that the BRCA1 is remarkably associated with ROS level. ${ }^{16,17)}$ Lovren et al. ${ }^{17)}$ demonstrated that BRCA1 had a protective effect against ROS generation. Therefore, the role of BRCA1 in paeonol treated cardiomyocytes were assessed.

Our results showed that paeonol improved the activity of radical scavenging enzymes, reduced ROS generation and further regulated Nod-like receptor protein 3 (NLRP3) inflammasome formation and nuclear factor-kappaB $(\mathrm{NF}-\kappa \mathrm{B})$ activity via a BRCA1 dependent manner following $\mathrm{A} / \mathrm{R}$ injury.

\section{Experimental}

Reagents Paeonol (purity $>99 \%$; HPLC) was purchased from desite biotech Co. (Chengdu, China). Rat cardiac myo- 
blast cells (H9C2) were obtained from the Cell Bank of the Chinese Academy of Sciences (Shanghai, China) and 3-7 passages of H9C2 cells were used in our research. Dulbecco's modified Eagle's medium (DMEM), L-glutamine and fetal bovine serum (FBS) were purchased from Invitrogen (Shanghai, China).

Cell Culture and Treatment $\mathrm{H} 9 \mathrm{C} 2$ cells were cultured in DMEM medium supplemented with $10 \% \mathrm{FBS}$ and $4 \mathrm{mM}$ glutamine at a $37^{\circ} \mathrm{C}$ humidified incubator with $5 \% \mathrm{CO}_{2}$. The cells model of $\mathrm{A} / \mathrm{R}$ injury was established as described in previous. ${ }^{18)}$ Briefly, the cells were grown under the hypoxic condition $\left(95 \% \mathrm{~N}_{2}, 5 \% \mathrm{CO}_{2}\right)$ for $2 \mathrm{~h}$, and then cultured in normoxia $\left(95 \% \mathrm{O}_{2}, 5 \% \mathrm{CO}_{2}\right)$ for $2 \mathrm{~h}^{19,20)}$ To evaluate the effect of paeonol on hypoxia-treated cells, H9C2 cells were pre-treated with paeonol $(10 \mu \mathrm{M})$ for $18 \mathrm{~h}$ prior to the induction of hypoxia. The total anti-oxidative capacity (T-AOC), lactate dehydrogenase (LDH) activity and creatine kinase (CK-MB) activity were assessed with commercial kits (H197, Jiancheng Biotech, Jiangsu, China). The level of NF- $\kappa \mathrm{B}$ activation was evaluated by TransAM p65 NF- $\kappa$ B assay kit (40096, Active Motif) following the instruction of the manufacturer. The level of BRCA1 mRNA and protein were determined using real-time PCR and Western blotting. The concentrations of interleukin 6 (IL-6), tumor necrosis factor (TNF)- $\alpha$ and IL- $1 \beta$ in medium were detected using enzyme-linked immunosorbent assay (ELISA) kit (Sizhengbai, Beijing, China).

Ethical Approval All data in our present study derive from H9C2 cell line that was purchased from the Cell Bank of the Chinese Academy of Sciences (Shanghai, China). So ethical permission was exempt.

BRCA1 Knockdown by Small Interfering RNA (siRNA) To investigate the function of BRCA1 in paeonol mediated antioxidant activity in $\mathrm{H} 9 \mathrm{C} 2$ cells, both BRCA1 siRNA and the control siRNA were transfected into H9C2 cells. SiRNA were purchased from Shanghai Bioegene Co., Ltd. After transfection of siRNA, cells were treated with paeonol and the induction of hypoxia as previously described. The efficacy of the siRNA interference was assessed by real-time PCR and Western blot.

Evaluation of ROS Generation and Activity of Antioxidant Enzymes ROS, T-AOC, superoxide dismutase (SOD) levels were examined using a commercial kit (Beyotime, Jiangsu, China, S0033S, S0121, S0101S). All experiments were performed as the manufacturer's instructions described. The level of ROS, T-AOC and SOD were determined by chemiluminiscence assay.

Cell Viability Determination Cell viability was measured by the ability to reduce 3-(4,5-dimethylthiazol-2-yl)-2,5diphenyltetrazolium bromide (MTT) (C0009, Beyotime, Jiangsu, China). H9C2 cells were seeded in 96-well plates and MTT reduction was evaluated by spectrophotometry using a microplate reader (Bio-Tek, U.S.A.).

Real-Time PCR Total RNA from H9C2 cells was extracted using TRIzol reagent (Invitrogen, Beijing, China). Two micrograms RNA was used to reverse transcribed to cDNA with a PrimeScript ${ }^{\mathrm{TM}}$ II 1st Strand cDNA Synthesis Kit (TaKaRa, 6210B, Dalian, China). cDNA was analyzed by Real-time PCR using ChamQ SYBR Color qPCR Master Mix (Vazyme, China, Q411-02) to detect the RNA expression level of BRCA1, NLRP3, ASC. The used primer sequences were as follows: BRCA1 (NCBI ID: 497672) sense: 5'-GTC AGC CCT
TGT TTGCAC TG-3'; BRCA1 antisense: 5'-TTG GAC CTT GGT GGT TGC TT-3'; NLRP3 sense: 5'-AAA GCC AAGAAT CCA CAGTGT AAC-3'; NLRP3 (NCBI ID: 287362) antisense: 5'TTGCCTCGC AGG TAA AGGT-3'; ASC (NCBI ID: 282817) sense: 5'-TTA TGGAAG AGT CTGGAGCTGTGG-3'; ASC antisense: 5'-AAT GAG TGC TTG CCT GTG TTGG-3'; GAPDH (NCBI ID: 24383) sense: 5'-TGA TGA CATCAA GAA GGT GGT GAA G-3'; antisense: 5'-TCC TTGGAG GCC ATG TGG GCCAT-3'; The RNA expression levels of these genes were presented as fold-change compared with control after normalization to glyceraldehyde-3-phosphate dehydrogenase (GAPDH) in each sample.

Western Blotting To evaluate the protein expression level of BRCA1, cells were incubated with a protein inhibitor and cell lysis buffer containing $50 \mathrm{~mm}$ Tris, $\mathrm{pH} 7.4,150 \mathrm{~mm} \mathrm{NaCl}$, $1 \%$ sodium deoxycholate, $1 \%$ Triton $\mathrm{X}-100,0.1 \%$ sodium dodecyl sulfate (SDS), $1 \mathrm{mM}$ phenylmethylsulfonyl fluoride (PMSF) for $1 \mathrm{~h}$ on ice. The cell lysate was centrifuged at $13000 \mathrm{~g}$ at $4{ }^{\circ} \mathrm{C}$ for $10 \mathrm{~min}$. The protein content of supernatants was detected using BCA kit (P0010, Beyotime). The total protein $(70 \mu \mathrm{g})$ mixed with loading buffer was heated at $100^{\circ} \mathrm{C}$ for $5 \mathrm{~min}$, separated by $8 \%$ SDS-polyacrylamide gel electrophoresis (PAGE) and then transferred to PVDF membranes (Millipore). The membranes were blocked using 5\% BSA and then exposed to diluted rabbit BRCA1 (1:1000, abcam, ab238983), NLRP3 (1:1000, abcam, ab214185), ACS (1:1000, abclonal, A1170) and GAPDH (1:1000, CST, 5174) overnight at $4{ }^{\circ} \mathrm{C}$. Then, membranes were washed 3 thimes with TBST and incubated with anti-rabbit immunoglobulin $\mathrm{G}$ (IgG) (Cell Signaling Technology, 7074) dilution for $2 \mathrm{~h}$. The chemiluminescence detection system was used to determinate the protein bands.

Statistical Analysis All results were presented as means \pm standard deviation (S.D.) Student's $t$-test was used for two groups comparison. One-way ANOVA was used for multiple groups comparison and Tukey's multiple comparisons test was used as post-hoc test in ANOVA. Analyses were performed by GraphPad Prism 8. $p$ Value less than 0.05 was considered a statistically significant difference.

\section{Results}

Pretreatment of Paeonol Protected against A/R Injury in H9C2 Cells Paeonol is a bioactive phenol and a white crystalline which is insoluble in water ${ }^{21)}$ (Fig. 1A). Firstly, in order to define the best time and best concentration of protective effects of paeonol, the time-course (Fig. 1B) study of paeonol were performed. We found that treatment of $\mathrm{H} 9 \mathrm{C} 2$ cells with paeonol $(10 \mu \mathrm{M})$ for $18 \mathrm{~h}$ before A/R could remarkably ameliorated cell viability. And the best concentration of $10 \mu \mathrm{M}$ was also confirmed by a concentration-dependent experiment (Fig. 1C). Further, we found pretreatment of $\mathrm{H} 9 \mathrm{C} 2$ cells with paeonol $(10 \mu \mathrm{M})$ for $18 \mathrm{~h}$ prior to the induction of $\mathrm{A} / \mathrm{R}$ distinctly reduced A/R-induced CK-MB (Fig. 1D) and LDH (Fig. 1E) concentration in the medium. Thus, we demonstrated that paeonol could alleviate A/R injury in H9C2 cells.

Pre-treat Paeonol Enhanced Antioxidant Enzymes Activity and Suppressed ROS Generation in $\mathrm{H} 9 \mathrm{C} 2$ Cells after A/R Injury Oxidative stress is considered as a vital mechanism of pathogenesis of $\mathrm{I} / \mathrm{R}$. We measured the ROS levels to evaluate the protective effects of paeonol. The results showed that the level of ROS after $\mathrm{A} / \mathrm{R}$ was more than twice as high 
A<smiles>COc1ccc(C(C)=O)c(O)c1</smiles>

B

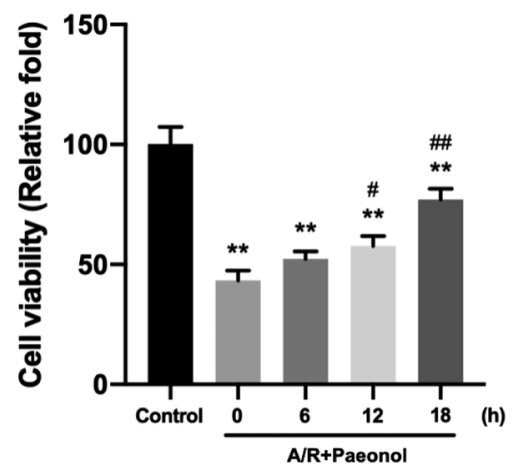

C

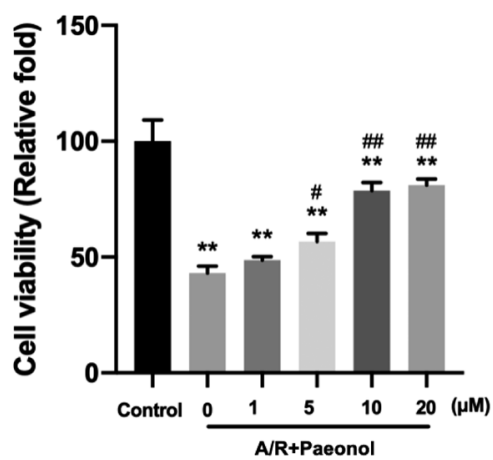

D

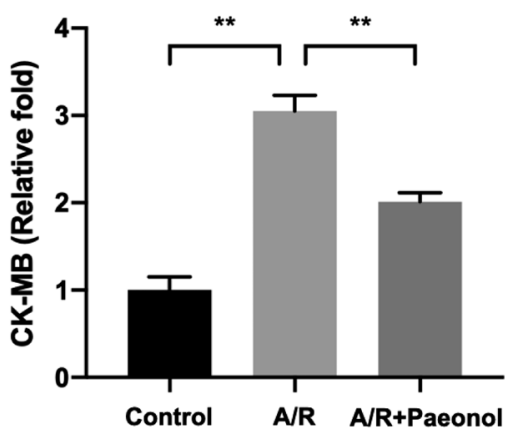

$E$

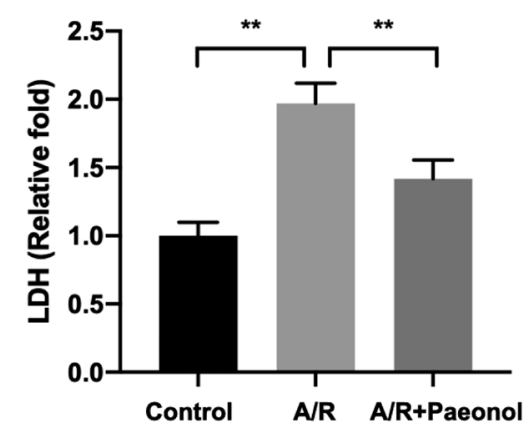

Fig. 1. Pretreatment of Paeonol Protected against A/R Injury in H9C2 Cells

$\mathrm{H} 9 \mathrm{C} 2$ cells were incubated with paeonol $(10 \mu \mathrm{M})$ for $18 \mathrm{~h}$ prior to $\mathrm{A} / \mathrm{R}$ injury. A, The structure of paeonol. B, the time-course experiment. compared with $0 \mathrm{~h}$ group, ${ }^{\#} p<0.05 ;{ }^{\# \#} p<0.01$. C , concentration-dependent experiment. D, relative fold of CK-MB concentration. E, relative fold of LDH activity. Results are presented as mean \pm standard deviation (S.D.) $n=3, * *<0.01$. One-way ANOVA was used in all experiments.

A

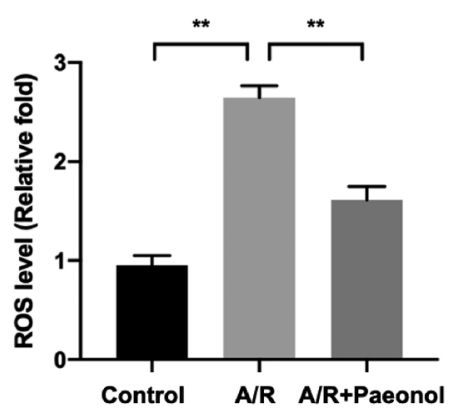

B

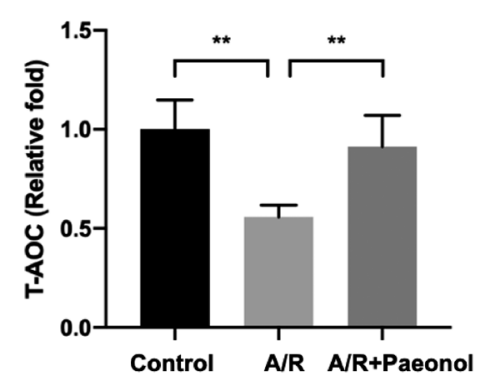

C

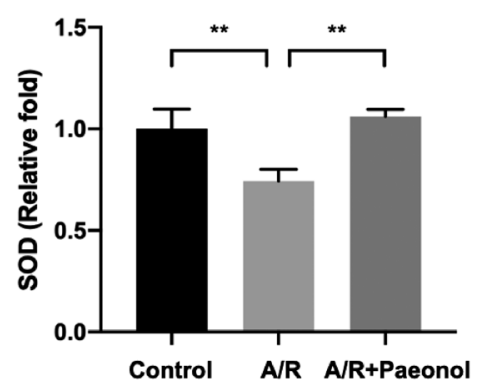

Fig. 2. Pre-treat Paeonol Enhanced Antioxidant Enzymes Activity and Suppressed ROS Generation in H9C2 Cells after A/R Injury

The $\mathrm{H} 9 \mathrm{C} 2$ cells were incubated with paeonol $(10 \mu \mathrm{M})$ for $18 \mathrm{~h}$ prior to the treatment of A/R. Level of H9C2 cells was measured by chemiluminiscence assay. A, relative fold of ROS level. B, relative fold of T-AOC. C, relative fold of SOD. Results are presented as mean \pm S.D. $n=3$, ** $p<0.01$. One-way ANOVA was used in all experiments.

as the control cells that were under normoxia condition and was remarkably inhibited by paeonol pre-treatment (Fig. 2A). In consistent with these results, paeonol dramatically enhanced the activity of T-AOC (Fig. 2B) and SOD (Fig. 2C) in H9C2 cells exposed to A/R.

Paeonol Improved the Expression of BRCA1 in $A / R$ Treated H9C2 Cells To verify the mechanism of the protective of paeonol, we detected the expression of BRCA1 mRNA and protein. We found that BRCA1 mRNA (Fig. 3A) and protein level (Fig. 3B) were significantly down-regulated after $\mathrm{A} / \mathrm{R}$ treatment than control group, while paeonol pre-treatment improved BRCA1 expression significantly. The results demonstrated that BRCA1 may be involved in the protective effect of paeonol.
Paeonol Alleviated A/R Injury in $\mathrm{H} 9 \mathrm{C} 2$ Cells via BRCA1-Dependent Manner To prove paeonol alleviated $\mathrm{A} / \mathrm{R}$ injury in $\mathrm{H} 9 \mathrm{C} 2$ cells via BRCA1-dependent manner, we established a short interfering RNA (siRNA) to target BRCA1 (siBRCA1) and verified that siBRCA1 markedly inhibited the expression of BRCA1 mRNA and protein in $\mathrm{H} 9 \mathrm{C} 2$ cells (Figs. 4A, B). We observed that BRCA1 knock-down could reverse the protective effect of paeonol in $\mathrm{A} / \mathrm{R}$ treated $\mathrm{H} 9 \mathrm{C} 2$ cells (Figs. 4C-E). These results indicated that paeonol ameliorate H9C2 cells injury after A/R treatment via a BRCA1 dependent manner.

BRCA1 Regulated ROS Level and Intracellular Antioxidant Capacity in Paeonol Pre-treated H9C2 Cells after A/R Injury Next, we demonstrated whether ROS level and 
A

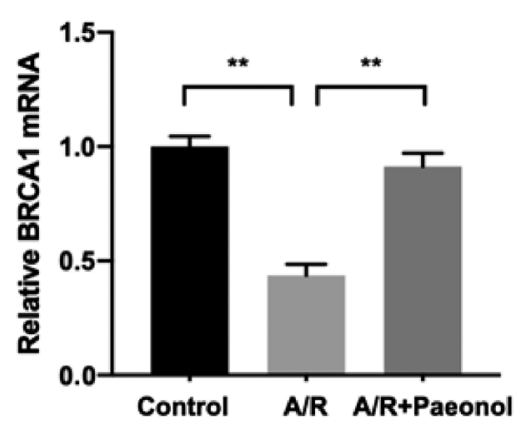

B

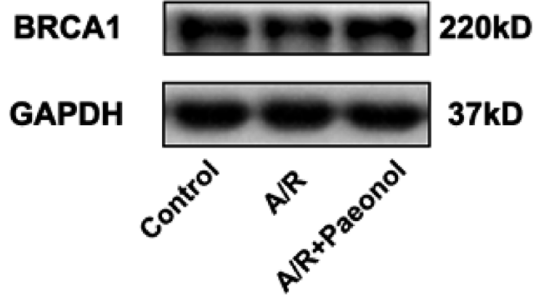

Fig. 3. Paeonol Improved the Expression of BRCA1 in A/R Treated H9C2 Cells

BRCA1 mRNA and protein level were measured by RT-qPCR and Western blot. A, relative fold of BRCA1 mRNA. B, The Western blot results of BRCA1 expression. Results are presented as mean \pm S.D. $n=3, * * p<0.01$. One-way ANOVA was used in all experiments.

A

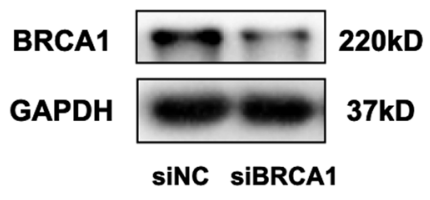

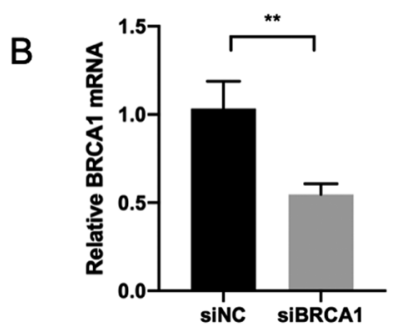

E

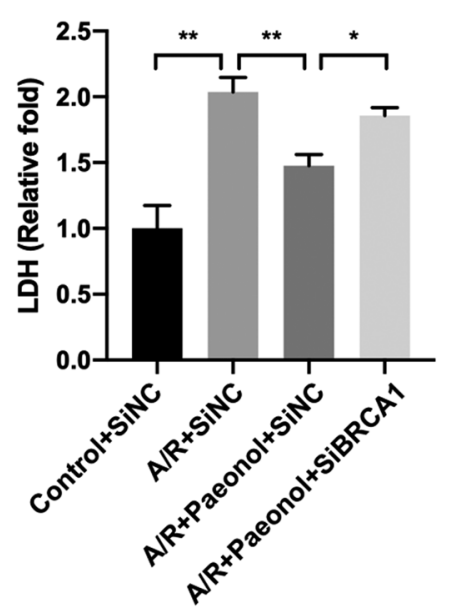

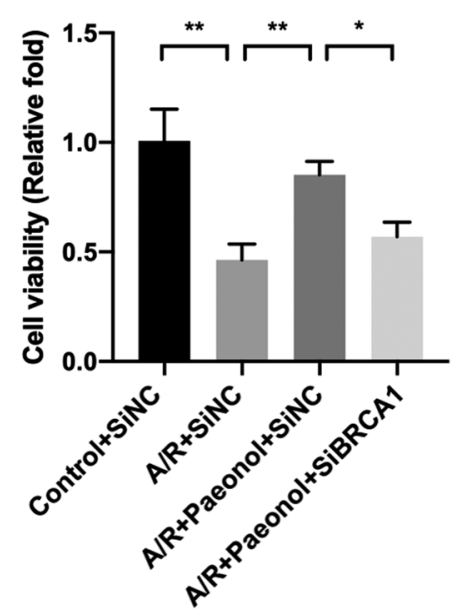

Fig. 4. Paeonol Alleviated A/R Injury in H9C2 Cells via BRCA1-Dependent Manner

BRCA1 expression after transfection of siNC or siBRCA1 was measured by WB and RT-qPCR. The CK-MB, LDH, and cell viability levels were determined. A, the Western blot results of BRCA1 expression. B, relative fold of BRCA1 mRNA. C, relative fold of CK-MB. D, relative fold of LDH. E, relative fold of cell viability. Results are presented as mean \pm S.D. $n=3, * * p<0.01, * p<0.05 . t$ test was used in 4B, and one-way ANOVA was used in 4C-4E.

intracellular antioxidant capacity were regulated by BRCA1. Chemiluminescence assay indicated that ROS generation was accelerated after $\mathrm{A} / \mathrm{R}$ treatment, and paeonol pre-treatment reduced ROS generation which were the same results as previous (Fig. 2A). however, ROS level improved after down-regulation of BRCA1 by siRNA (Fig. 5A). Meanwhile, we found T-AOC (Fig. 5B) and SOD (Fig. 5C) levels decreased after down-regulation of BRCA1.

BRCA1 Regulated NLRP3 Inflammasome Pathway and NF- $\kappa$ B Pathway in Paeonol Pre-treated H9C2 Cells after A/R Injury To further understand the mechanism of paeonol which exerted a protective effcet on $A / R$ injury, we observed the variation of NLRP3 inflammasome pathway and found that NLRP3 inflammasome formation markers (NLRP3, ACS) and IL- $1 \beta$ secretion were promoted after A/R treatment while paeonol pre-treatment suppressed their expressions (Figs. 6A-D). Moreover, A/R condition and paeonol pretreatment played a similar role in NF- $\kappa$ B/TNF- $\alpha /$ IL- 6 pathway (Figs. 6E-G). However, transfection of siBRCA1 could reverse the effects of down-regulation of NLRP3 inflammasome pathway and NF- $\kappa$ B pathway by pre-treatment of paeonol. These data suggested that paeonol conducted its protective effect by regulating NLRP3 inflammasome pathway and NF- $\kappa \mathrm{B}$ pathway via a BRCA1 dependent manner. NLRP3 inflammasome 
A

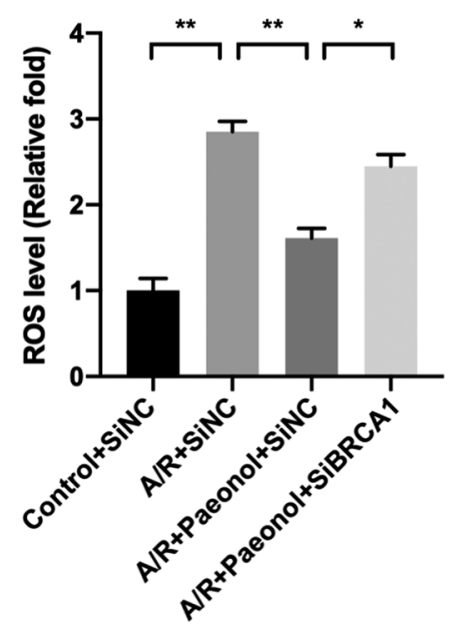

B

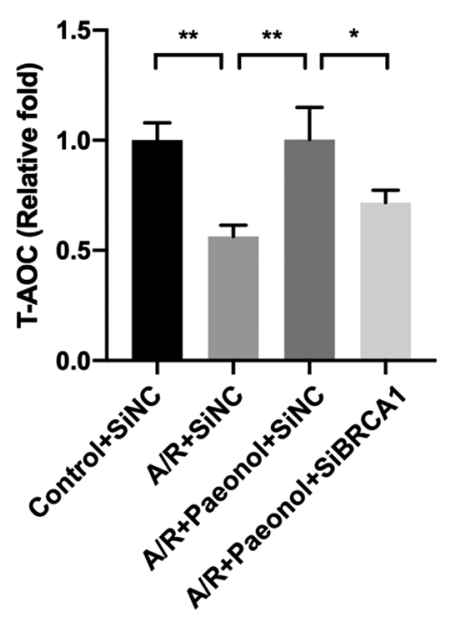

C

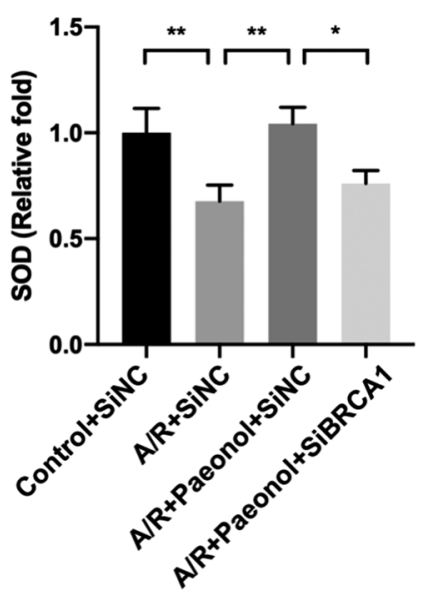

Fig. 5. BRCA1 Regulated ROS Level and Intracellular Antioxidant Capacity in Paeonol Pre-treated H9C2 Cells after A/R Injury

After pre-treatment of paeonol, $\mathrm{H} 9 \mathrm{C} 2$ cells were exposed to A/R injury. ROS, T-AOC, SOD levels were determined by chemiluminescence assay. A, relative fold of ROS level. B, relative fold of T-AOC. C, relative fold of SOD. Results are presented as mean \pm S.D. $n=3,{ }^{* *} p<0.01,{ }^{*} p<0.05$. One-way ANOVA was used in all experiments.

A

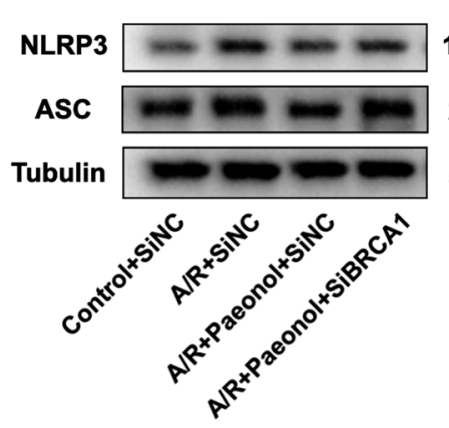

$\mathrm{E}$

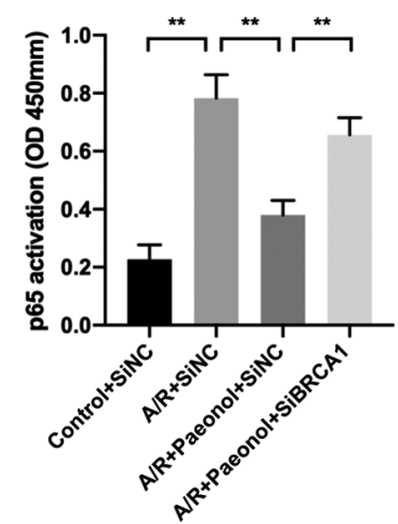

B

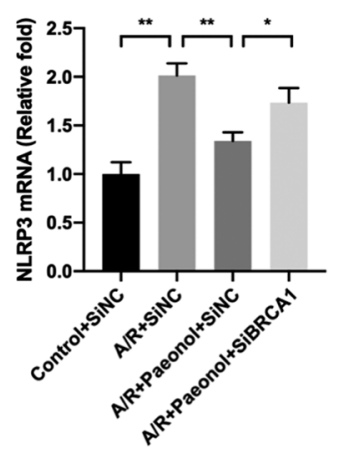

$\mathrm{F}$

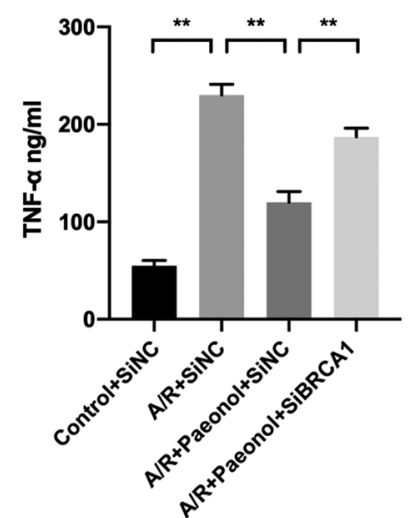

C

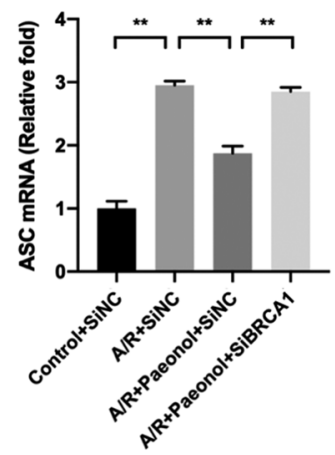

D

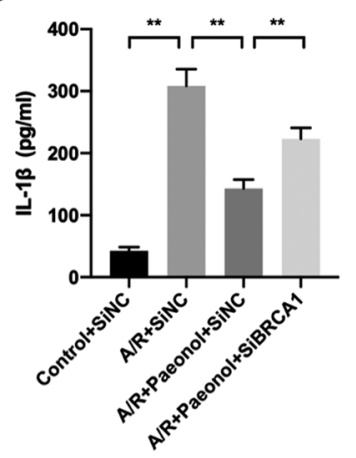

G

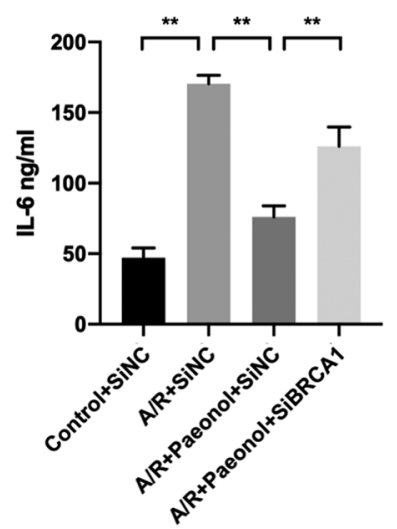

Fig. 6. BRCA1 Regulated NLRP3 Inflammasome Pathway and NF- $\kappa$ B Pathway in Paeonol Pre-treated H9C2 Cells after A/R Injury

After pre-treatment of paeonol, H9C2 cells were exposed to A/R injury. NLRP3, ASC mRNA and protein levels were determined by qRT-PCR and Western blotting. NF- $\kappa$ B activation was evaluated by TransAM p 65 NF- $\kappa$ B assay kit. The concentrations of IL-6, TNF- $\alpha$ and IL-1 $\beta$ in medium were detected using ELISA kit. A, NLRP3 and ACS protein levels. B-C, NLRP3 and ACS mRNA expression levels. D, IL-1 $\beta$ level. E, p65 activation level. F, TNF- $\alpha$ level. G, IL-6 level. Results are presented as mean \pm S.D. $n=3, * * p<0.01, * p<0.05$. One-way ANOVA was used in all experiments.

and NF- $\kappa \mathrm{B}$ are all extremely sensitive to the oxidative stress, and we have demonstrated BRCA1 regulated ROS level and intracellular antioxidant capacity in paeonol pre-treated H9C2 cells. Therefore, we deduced paeonol alleviated A/R injury via $\mathrm{BRCA} 1 / \mathrm{ROS} / \mathrm{NLRP} 3$ and $\mathrm{BRCA} 1 / \mathrm{ROS} / \mathrm{NF}-\kappa \mathrm{B}$ pathway in H9C2 cells (Fig. 7).

\section{Discussion}

Paeonol is a phenolic compound obtained from cortex moutan which has been broadly used as a traditional Chinese herb in prevention and treatment of many diseases including, rheumatoid arthritis, hepatitis, cerebral ischemic injury and acute kidney injury., ${ }^{9,19)}$ Recent studies suggest paeonol could exert protective roles in the disease of cardiovascular system, ${ }^{20,21)}$ 


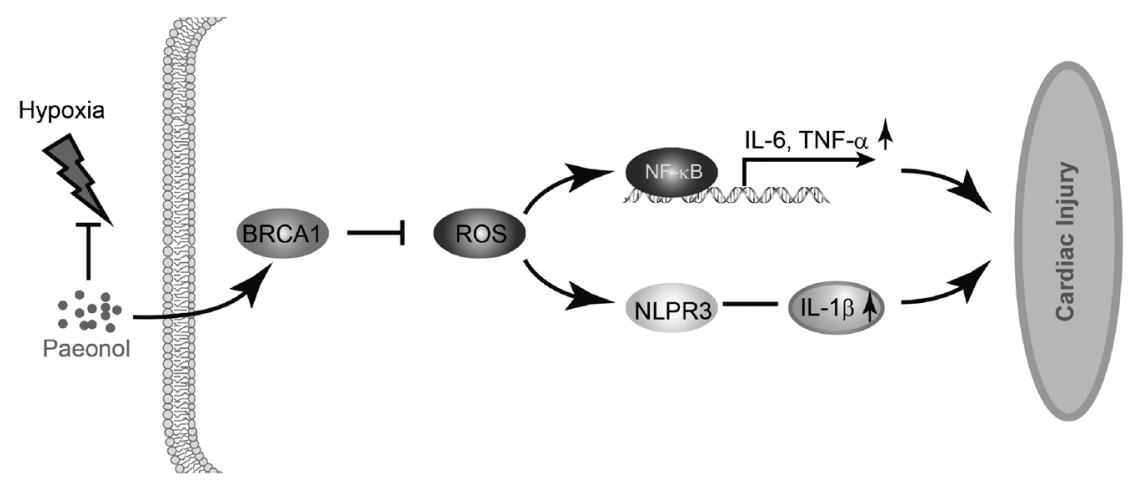

Fig. 7. Paeonol Alleviated A/R Injury in $\mathrm{H} 9 \mathrm{C} 2$ Cells via $\mathrm{BRCA} / \mathrm{ROS} / \mathrm{NLRP} 3$ and BRCA1/ROS/NF- $\kappa \mathrm{B}$ Pathway

which is consistent with our results derived from A/R injury experiments in $\mathrm{H} 9 \mathrm{C} 2$ cells. Our results indicated that paeonol markedly resisted the releasing of $\mathrm{LDH}$ and $\mathrm{CK}-\mathrm{MB}$ in $\mathrm{A} / \mathrm{R}$ treated H9C2 cells.

In the past years, many literatures have reported that paeonol displays considering pharmacological properties for inhibition of tumor grown, ${ }^{22,23)}$ alleviating inflammatory response, ${ }^{10,24)}$ mediating oxidative stress and modulating the immune system. ${ }^{25)}$ However, the effective physiological concentration of paeonol in human are still unknown. It is wildly believed that $20-200 \mathrm{mg} / \mathrm{kg}$ concentration of paeonol possesses therapeutic effect in mice and rats experiments. ${ }^{26)}$ In our study, $10 \mu \mathrm{M}$ was determined as the best concentration to produce the protective effect in vitro. Previous studies have clearly demonstrated that paeonol can interact with many molecular targets including pro-inflammatory mediators, enzymes and proteins, such as IL-6, HDAC3, TLR, Smad3. ${ }^{27)}$ However, which is the primary target of paeonol is still unclear. In this study, we found paeonol regulated BRCA1 expression, though the underlying mechanism still remains to be determined.

BRCA1 is a tumor suppressor which is wildly mutated in breast cancer. We found that paeonol alleviated cardiac injury via BRCA1 dependent manner. BRCA1 is reported to induce antioxidant gene expression and resistance to oxidative stress. $^{28)}$ In addition, BRCA1 was confirmed to regulate nuclear factor-E2-related factor 2 (Nrf2)-dependent antioxidant signaling by physically interacting with Nrf2 and promoting its stability and activation. ${ }^{29)}$ We demonstrated down regulation of BRCA1 promoted ROS production and repressed intracellular antioxidant capacity represented by T-AOC and SOD.

NLRP3 inflammasome consists of NLRP3, ACS and procaspase- $1,{ }^{30)}$ and its activation is composed of two steps which contain NLRP3 inflammasome information and IL- $1 \beta$ secretion. ${ }^{31)} \mathrm{NF}-\kappa \mathrm{B}$ is a redox-sensitive transcription factor. Tremendous studies have demonstrated that ROS generation stimulate NLRP3 inflammasome and NF- $\kappa$ B activation ${ }^{32-34)}$ which both are pivotal factors for myocardial I/R injury. Therefore, these pathways were assessed. As expected, down regulation of BRCA1 enhanced NLRP3, ACS expression and IL- $1 \beta$ secretion. Further, down regulation of BRCA1 increased NF- $\kappa$ B activation and TNF- $\alpha$, IL- 6 secretion in medium. The increasing activation of these pathways destroyed paeonol induced protective effect. We concluded Paeonol alleviated $\mathrm{A} / \mathrm{R}$ injury in $\mathrm{H} 9 \mathrm{C} 2$ cells via $\mathrm{BRCA} 1 / \mathrm{ROS} / \mathrm{NLRP} 3$ and BRCA1/ROS/NF- $\kappa$ B pathway.

In summary, we confirmed that paeonol ameliorate $A / R$ in- jury in H9C2 cells via BRCA1/ROS regulated NLRP3 inflammasome and NF- $\kappa \mathrm{B}$ activation pathway (as expressed in Fig. 7). These results offers promise for the application of paeonol as a cardioprotectant in $\mathrm{A} / \mathrm{R}$ (IR) injury. A follow-up study is warranted to define the underlying mechanism of BRCA1 expression induced by paeonol.

Conflict of Interest The authors declare no conflict of interest.

\section{References}

1) Long T. Y., Jing R., Kuang F., Huang L., Qian Z. X., Yang T. L., Braz. J. Med. Biol. Res., 50, e5861 (2017).

2) Liu H., Jing X., Dong A., Bai B., Wang H., Cell. Physiol. Biochem., 44, 1011-1023 (2017)

3) Hentia C., Rizzato A., Camporesi E., Yang Z., Muntean D. M., Săndesc D., Bosco G., Brain and Behavior, 8, e00959 (2018).

4) Tullio F., Penna C., Cabiale K., Femmino S., Galloni M., Pagliaro P., Biomed. Pharmacother., 90, 194-202 (2017).

5) Kan C., Ungelenk L., Lupp A., Dirsch O., Dahmen U., Transplantation, 102, 368-377 (2018).

6) Cursio R., Colosetti P., Gugenheim J., BioMed Research International, 2015, 417590 (2015).

7) Bagheri F., Khori V., Alizadeh A. M., Khalighfard S., Khodayari S., Khodayari H., Life Sci., 165, 43-55 (2016).

8) Misra M. K., Sarwat M., Bhakuni P., Tuteja R., Tuteja N., Med. Sci. Monit., 15, RA209-RA219 (2009).

9) Fan H.-Y., Qi D., Yu C., Zhao F., Liu T., Zhang Z. K., Yang M. Y., Zhang L. M., Chen D. Q., Du Y., Oncotarget., 7, 39497-39510 (2016).

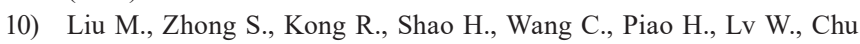
X., Zhao Y., Biomed. Pharmacother., 95, 914-921 (2017).

11) Choy K. W., Lau Y. S., Murugan D., Mustafa M. R., PLOS ONE, 12, e0178365 (2017).

12) Zhao Y., Fu B., Zhang X., Zhao T., Chen L., Zhang J., Wang X., Brain Res. Bull., 109, 61-67 (2014).

13) Shukla P. C., Singh K. K., Quan A., Al-Omran M., Teoh H., Lovren F., Cao L., Rovira I. I., Pan Y., Brezden-Masley C., Yanagawa B., Gupta A., Deng C. X., Coles J. G., Leong-Poi H., Stanford W. L., Parker T. G., Schneider M. D., Finkel T., Verma S., Nat. Commun., 2,1-11 (2011).

14) Leung J. W., Makharashvili N., Agarwal P., Chiu L. Y., Pourpre R. Cammarata M. B., Cannon J. R., Sherker A., Durocher D., Brodbelt J. S., Paull T. T., Miller K. M., Genes Dev., 31, 260-274 (2017).

15) Chiang H. C., Zhang X., Li J., Zhao X., Chen J., Wang H. T., Jatoi I., Brenner A., Hu Y., Li R., Nucleic Acids Res., 47, 5086-5099 (2019)

16) Kang H. J., Hong Y. B., Kim H. J., Wang A., Bae I., Toxicol. Lett., 209, 154-160 (2012). 
17) Lovren F., Pan Y., Quan A., Singh K. K., Khan R., Gupta N., Brezden-Masley C., Teoh H., Wheatcroft M. D., Al-Omran M., Verma S., J. Thorac. Cardiovasc. Surg., 147, 1946-1955.e1 (2014).

18) Ding Y., Li Q., Xu Y., Chen Y., Deng Y., Zhi F., Qian K., PLOS $O N E$, 11, e0154375 (2016).

19) Liu N., Feng X., Wang W., Zhao X., Li X., Inflamm. Res., 66, 603-610 (2017)

20) Lu L., Qin Y., Chen C., Guo X., Oxid. Med. Cell. Longev., 2018, 1098617 (2018).

21) Zhang L., Ma C., Gu R., Zhang M., Wang X., Yang L., Liu Y., Zhou Y., He S., Zhu D., Eur. J. Pharmacol., 834, 257-265 (2018).

22) Li H., Wang S., Zhang B., Xie Y., Wang J., Yang Q., Cao W., Hu J., Duan L., Planta Med., 78, 135-140 (2012).

23) Zhang L., Tao L., Shi T., Zhang F., Sheng X., Cao Y., Zheng S., Wang A., Qian W., Jiang L., Lu Y., IUBMB Life, 67, 778-788 (2015).

24) Saahene R. O., Wang J., Wang M. L., Agbo E., Pang D., Cancer Biother. Radiopharm., 33, 233-240 (2018).

25) He L. X., Tong X., Zeng J., Tu Y., Wu S., Li M., Deng H., Zhu M., Li X., Nie H., Yang L., Huang F., Inflammation, 39, 1904-1917 (2016).
26) Adki K. M., Kulkarni Y. A., Life Sci., 250, 117544 (2020).

27) Zhang L., Li D., Liu L., Int. Immunopharmacol., 72, 413-421 (2019).

28) Gorrini C., Baniasadi P. S., Harris I. S., et al., J. Exp. Med., 210, 1529-1544 (2013)

29) Bae I., Fan S., Meng Q., Rih J. K., Kim H. J., Kang H. J., Xu J., Goldberg I. D., Jaiswal A. K., Rosen E. M., Cancer Res., 64, 78937909 (2004).

30) He Y., Hara H., Núñez G., Trends Biochem. Sci., 41, 1012-1021 (2016).

31) Zhang Z., Duan Q., Zhao H., Liu T., Wu H., Shen Q., Wang C., Yin T., Cancer Lett., 382, 53-63 (2016).

32) Kanzaki H., Shinohara F., Kanako I., Yamaguchi Y., Fukaya S., Miyamoto Y., Wada S., Nakamura Y., Redox Biology., 8, 186-191 (2016).

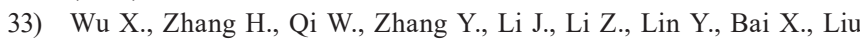
X., Chen X., Yang H., Xu C., Zhang Y., Yang B., Cell Death Dis., 9 , 171 (2018).

34) Deng X., Huang W., Peng J., Zhu T. T., Sun X. L., Zhou X. Y., Yang H., Xiong J. F., He H. Q., Xu Y. H., He Y. Z., Inflammation, 41, 260-275 (2018). 